\title{
BIOMETRIA DE EQÜINOS DA RAÇA CRIOULA NO BRASIL
}

(Biometric in brazilian criollo horse breed)

\author{
KURTZ FILHO, M.; LÖF, H.K. \\ Departamento de Morfologia, Centro de Ciências da Saúde (CCS), Universidade Federal de Santa Maria (UFSM)-RS, Brasil. \\ Curso de Medicina Veterinária, Centro de Ciências Rurais (CCR), UFSM, Santa Maria, RS, Brasil.
}

\begin{abstract}
RESUMO-Este trabalho teve o objetivo de avaliar as medidas biométricas de eqüinos da raça crioula criados no Brasil. Os dados relativos à altura, perímetros torácico e de canela de 2.054 eqüinos (866 machos e 1.188 fêmeas), com idade entre dois e 13 anos, apresentados na Exposição anual de Esteio, RS, para concurso de avaliação morfológica, no período de agosto de 1993 a agosto de 2002 foram analisados. Nos machos a altura média foi de $1,43 \mathrm{~m}(0,02)$, perímetro torácico de $1,79 \mathrm{~m}(0,06)$ e perímetro da canela de $0,20 \mathrm{~m}(0,01)$; para as fêmeas verificou-se uma altura média de $1,42 \mathrm{~m}(0,02)$, perímetro torácico de $1,80 \mathrm{~m}(0,05)$ e perímetro da canela de $0,19 \mathrm{~m}(0,01)$. Os parâmetros analisados estiveram dentro do padrão determinado pela Associação Brasileira de Criadores de Cavalos Crioulos, com tendência à diminuição da altura.
\end{abstract}

Palavras-chave: eqüinos crioulos; altura; perímetro; tórax.

ABSTRACT- In order to evaluate the biometric values of 2.054 - 866 males and 1.188 females - Brazilian native horse breed,aging between 2 and 13 years old, height, chest girth and cannon bone girth measurements were performed and analyzed. The horses were exposed at Esteio Horse Show Rio Grande do sul Brazil, for confarmation e evaluation, during August 1993 to August 2002. The whole average were height $1.43 \mathrm{~m}(0.02)$, chest girth $1.79 \mathrm{~m}(0.06)$ and cannon bone girth $0.20 \mathrm{~m}(0.01)$; for males, and height $1.42 \mathrm{~m}(0.02)$, chest girth $1.80 \mathrm{~m}(0.05)$ and cannon bone girth $0.19 \mathrm{~m}(0.01)$ for females. It has been observed that all the parameters are within standard values from breeders associations besides an observed tendency for diminished height.

Key words: criollo horse; height; chest girth.

\section{INTRODUÇÃO}

Os cavalos chegaram ao continente americano ao final do século $\mathrm{XV}$, originários da península ibérica, território que sofreu conquista e dominação por diferentes povos. Na América estes eqüinos espalharam-se a partir do Panamá, Colômbia, região do rio da Prata e Paraguai formando grandes manadas (ASSUNÇÃO, 2000). No final do século XIX e início do século XX, sobre os descendentes destes cavalos de origem européia, vivendo agora no continente americano, criadores argentinos, uruguaios e brasileiros iniciaram a recuperação e seleção dos animais para formação da raça crioula.

Posteriormente, formaram-se nestes países associações de criadores de cavalos da raça crioula e estas, na tentativa de fixar um padrão racial comum determinaram medidas de altura, perímetros torácico e de canela. Ressalta-se que os criadores fizeram também uma seleção funcional. Assim, animais excepcionais funcionalmente, mas com conformação morfológica inadequada para o padrão racial provavelmente foram aceitos (DOWDALL, 2003). Ao final da década de 50 as associações argentina, brasileira, chilena (no Chile o cavalo de origem e morfologicamente semelhante ao crioulo, é denominado cavalo chileno), uruguaia e posteriormente a paraguaia aprovaram o padrão racial para o cavalo crioulo: um cavalo de perfil reto ou subconvexo, eumétrico e mesomorfo, com medidas determinadas (DOWDALL, 2003).

Estas medidas são utilizadas com pequenas variações entre as associações, pois se permite uma flutuação conveniente em função da atividade laboral ou esportiva distinta de cada país. A altura ideal determinada para os machos foi de $1,44 \mathrm{~m}$, com amplitude de $1,38 \mathrm{~m}$ a $1,50 \mathrm{~m}$, recomendando-se, entretanto, não ultrapassar $1,48 \mathrm{~m}$; para as fêmeas: $2 \mathrm{~cm}$ a menos. O perímetro torácico ideal para os machos seria de $1,78 \mathrm{~m}$, para as fêmeas $2 \mathrm{~cm}$ a mais. O perímetro da canela para os machos seria de $0,19 \mathrm{~m}$ e para as fêmeas $1 \mathrm{~cm}$ a menos (DOWDALL, 1985). 


\section{KURTZ e LÖF}

As medidas adotadas pela Associação Brasileira de Criadores de Cavalos Crioulos (ABCCC) para os machos são: altura entre 1,40 e $1,50 \mathrm{~m}$; perímetro torácico mínimo de $1,68 \mathrm{~m}$; e perímetro de canela mínimo de $0,18 \mathrm{~m}$. Para as fêmeas as medidas são: altura entre 1,38 e 1,50m; perímetro torácico mínimo de $1,70 \mathrm{~m}$; e perímetro da canela mínimo de $0,17 \mathrm{~m}$, (ASSOCIAÇÃO BRASILEIRA DE CRIADORES DE CAVALOS CRIOULOS, 2006).

A raça crioula no Brasil possui ativo mercado de leilões de animais, e um studbook expressivo, com grande número de animais nascidos e inscritos anualmente. Deve-se ressaltar que são ainda raros os trabalhos científicos com esta raça, e dados biométricos estatísticos no Brasil são inexistentes. O mercado, a indústria do cavalo por si justifica a realização de pesquisas na área, pois é atividade que envolve diferentes segmentos da sociedade, com significativo impacto econômico. Após mais de 70 anos de seleção da raça crioula no Brasil, é necessária a produção de ferramentas que auxiliem a criação. Este trabalho teve o objetivo de analisar as medidas específicas da biometria da raça e o seu enquadramento no padrão determinado pela associação de criadores, nos animais apresentados no evento morfológico de maior importância para a raça crioula no Brasil.

\section{MATERIAL E MÉTODO}

Foram avaliados 2.054 eqüinos (866 machos e 1.188 fêmeas), levados à Exposição de Esteio, RS, no período de agosto 1993 a agosto de 2002, com idade superior a dois anos e com até 13 anos de idade, para apresentação em concurso de avaliação morfológica (conformação), sendo uma amostragem significativa do estágio seletivo dos animais brasileiros, representantes das diferentes linhagens da raça crioula. De todos os animais foram tomadas as seguintes medidas (em unidades métricas): Altura da cernelha, verificada com hipômetro, tomada do ponto mais alto da região interescapular, e sua distância do solo com ferraduras; perímetro torácico, verificado com fita métrica, na porção mais estreita do tórax, caudalmente à cernelha, na porção dorsal das últimas vértebras torácicas e ventralmente no terço caudal do esterno; perímetro da canela, verificado com fita métrica, relativo ao contorno do membro torácico esquerdo, no seu terço médio.

Estas medidas foram tomadas pelos técnicos da Associação Brasileira de Criadores de Cavalos Crioulos, em planilha da ABCCC. Os dados foram analisados usando o programa estatístico SAS (SAS, 1999) utilizando a análise da variância e teste de Tukey, com nível de significância de 0,05.

\section{RESULTADOS E DISCUSSÃO}

Os resultados demonstram que na média, os três parâmetros analisados estão dentro do padrão morfológico determinado tanto pelo padrão da ABCCC, como pelo padrão comum das demais associações. Em todos os parâmetros analisados há diferença significativa entre os sexos $(p<0,05)$ e não há influências significativas dos anos de nascimento ou de exposição. Evidencia-se que o parâmetro altura (TABELA 1), de acordo com DOWDALL (1985) está num limite inferior ao padrão acertado pelas demais associações latinoamericanas.

TABELA1- VALORES ABSOLUTOS DA ALTURA MÉDIA, PERÍMETRO TORÁCICO MÉDIO E PERÍMETRO MÉDIO DA CANELA DE EQÜINOS CRIOULOS, NOS PARTICIPANTES DA EXPOSIÇÃO DE ESTEIO, NO PERÍODO DEAGOSTO DE 1993AAGOSTO DE 2002.

\begin{tabular}{|c|c|c|c|c|c|c|c|c|}
\hline \multirow[b]{2}{*}{ PARÂMETRO (m) } & \multicolumn{4}{|c|}{ MACHOS } & \multicolumn{4}{|c|}{ FÊMEAS } \\
\hline & NÚMERO & $\begin{array}{c}\text { MÉDIA } \\
\text { (M) }\end{array}$ & $\begin{array}{l}\text { DESVIO } \\
\text { PADRÃO }\end{array}$ & $\begin{array}{l}\text { AMPLITUDE } \\
(\mathrm{M})\end{array}$ & NÚMERO & $\begin{array}{c}\text { MÉDIA } \\
\text { (M) }\end{array}$ & $\begin{array}{l}\text { DESVIO } \\
\text { PADRÃO }\end{array}$ & $\begin{array}{l}\text { AMPLITUDE } \\
\text { (M) }\end{array}$ \\
\hline altura & 866 & 1,43 & 0,02 & $1,40-1,49$ & 1.188 & 1,42 & 0,02 & $1,38-1,48$ \\
\hline perímetro torácico & 866 & 1.79 & 0.06 & $1.68-1.96$ & 1.188 & 1.80 & 0.05 & $1.68-1.98$ \\
\hline canela & 866 & 0.20 & 0.01 & $0.18-0.22$ & 1.188 & 0.19 & 0.01 & $0.17-0.21$ \\
\hline
\end{tabular}


Biometria de eqüinos da raça crioula no brasil.

Estudos semelhantes existem em outras raças brasileiras de sela: como na raça mangalarga marchador na qual CABRAL et al. (2004) encontraram os seguintes valores: a altura dos machos foi de $1,515 \mathrm{~m}$, para as fêmeas: $1,516 \mathrm{~m}$; perímetro torácico para os machos: $1,808 \mathrm{~m}$, para as fêmeas 1,757; perímetro da canela para os machos: $0,197 \mathrm{~m}$ e para as fêmeas: 0,190 , valores que se enquadram nos padrões recomendados pela associação de criadores da raça mangalarga marchador, que seleciona animais de estatura maior se comparada à raça crioula. Em outras raças brasileiras os valores são próximos aos observados na raça crioula: segundo McMANUS et al. (2005), na raça campeira, com origem semelhante à crioula, as médias sem considerar o sexo foram: altura: $1,44 \mathrm{~m}$; perímetro torácico: $1,73 \mathrm{~m}$ e perímetro de canela: $0,18 \mathrm{~m}$; na raça pantaneira, raça sobre a qual existe razoável bibliografia e também de origem semelhante à crioula a altura média dos machos foi de $1,416 \mathrm{~m}$ e das fêmeas $1,368 \mathrm{~m}$ (MISERANI et al., 2002).

Na TABELA 2 os resultados são apresentados de acordo com a denominação etária preconizada pela associação de criadores. Verificou-se nos machos uma maior homogeneidade de medidas do que nas fêmeas. Isto se deve provavelmente, ao processo de seleção dos machos, os quais, para a obtenção do certificado de registro definitivo, são submetidos a avaliações morfológicas prévias, procedimento que não é realizado nas fêmeas.

TABELA2 - VALORES ABSOLUTOS DAALTURA MÉDIA, PERÍMETRO TORÁCICO MÉDIO E PERÍMETRO MÉDIO DA CANELA (EM METROS: M) DE EQÜINOS CRIOULOS, REPRESENTADOS EM CATEGORIAS*: POTRANCO MENOR (1), POTRANCO MAIOR (2), CAVALO MENOR (3), CAVALO ADULTO (4), POTRANCA MENOR (5), POTRANCA MAIOR (6), ÉGUA MENOR (7), ÉGUA ADULTA (8), NOS PARTICIPANTES DAEXPOSIÇÃO DE ESTEIO, NO PERIODO DEAGOSTO DE 1993AAGOSTO DE 2002.

\begin{tabular}{|c|c|c|c|c|c|c|c|c|c|}
\hline \multicolumn{5}{|c|}{ MACHOS } & \multicolumn{5}{|c|}{ FÊMEAS } \\
\hline CATEGORIA & NÚMERO & $\begin{array}{l}\text { ALTURA } \\
(\mathrm{m})\end{array}$ & $\begin{array}{c}\text { PERÍMETRO } \\
\text { TORÁCICO } \\
(\mathrm{m})\end{array}$ & $\begin{array}{c}\text { CANELA } \\
(\mathrm{m})\end{array}$ & CATEGORIA & NÚMERO & $\begin{array}{l}\text { ALTURA } \\
(\mathrm{m})\end{array}$ & $\begin{array}{l}\text { PERÍMETRO } \\
\text { TORÁCICO(m) }\end{array}$ & CANELA (m) \\
\hline 1 & 95 & 1,426 & $1,780^{b}$ & $0,193^{c}$ & 5 & 173 & $1,412^{b}$ & $1,789^{C}$ & $0,186^{b c}$ \\
\hline 2 & 92 & 1,429 & $1,802^{a}$ & $0,195^{b c}$ & 6 & 176 & $1,410^{b}$ & $1,799^{b c}$ & $0,185^{\mathrm{c}}$ \\
\hline 3 & 207 & 1,428 & $1,799^{a}$ & $0,196^{b}$ & 7 & 356 & $1,418^{a}$ & $1,812^{a}$ & $0,187^{b}$ \\
\hline 4 & 352 & 1,431 & $1,789^{\mathrm{ab}}$ & $0,199^{a}$ & 8 & 458 & $1,420^{a}$ & $1,805^{a b}$ & $0,189^{a}$ \\
\hline
\end{tabular}

Na coluna, médias com a letra diferente diferem estatisticamente pelo Teste de Tukey.

*Potranco(a) menor: de 31 até 33 meses.

Potranco(a) maior: de 34 até 37 meses.

Cavalo (égua) menor: de 38 até 49 meses.

Cavalo (égua) adulto(a): de 50 até 145 meses.

TABELA3- $\quad$ ALTURA MÉDIA, PERÍMETRO TORÁCICO MÉDIO E PERÍMETRO MÉDIO DA CANELA (EM METROS: M) DE EQÜINOS CRIOULOS, DIVIDOS POR SEXO, NAS RESPECTIVAS IDADES, NOS PARTICIPANTES DAEXPOSIÇÃO DE ESTEIO, NO PERIODO DEAGOSTO DE 1993AAGOSTO DE 2002.

\begin{tabular}{|c|c|c|c|c|c|c|c|c|}
\hline \multirow[b]{2}{*}{$\begin{array}{r}\text { IDADE } \\
\text { (ANOS) }\end{array}$} & \multicolumn{4}{|c|}{ MACHOS } & \multicolumn{4}{|c|}{ FÊMEAS } \\
\hline & NÚMERO & $\begin{array}{l}\text { ALTURA } \\
(\mathrm{m})\end{array}$ & $\begin{array}{c}\text { PERÍMETRO } \\
\text { TORÁCICO } \\
(\mathrm{m}) \\
\end{array}$ & CANELA $(\mathrm{m})$ & NÚMERO & $\begin{array}{l}\text { ALTURA } \\
(\mathrm{m})\end{array}$ & $\begin{array}{c}\text { PERÍMETRO } \\
\text { TORÁCICO } \\
(\mathrm{m}) \\
\end{array}$ & CANELA $(m)$ \\
\hline 2 & 15 & 1,424 & $1,770^{\mathrm{ab}}$ & $0,194^{c}$ & 15 & 1,407 & 1,772 & $0,183^{b}$ \\
\hline 3 & 203 & 1,427 & $1,794^{a b}$ & $0,195^{\mathrm{bc}}$ & 379 & 1,411 & 1,793 & $0,186^{b}$ \\
\hline 4 & 194 & 1,431 & $1,800^{a b}$ & $0,197^{a b c}$ & 345 & 1,420 & 1,817 & $0,187^{a b}$ \\
\hline 5 & 124 & 1,434 & $1,805^{a}$ & $0,198^{a b c}$ & 193 & 1,422 & 1,815 & $0,188^{a b}$ \\
\hline 6 & 78 & 1,428 & $1,776^{\mathrm{ab}}$ & $0,200^{a b c}$ & 93 & 1,422 & 1,799 & $0,189^{\mathrm{ab}}$ \\
\hline 7 & 51 & 1,429 & $1,779^{a b}$ & $0,200^{a b c}$ & 61 & 1,422 & 1,796 & $0,189^{a b}$ \\
\hline 8 & 33 & 1,430 & $1,775^{\mathrm{ab}}$ & $0,201^{a b}$ & 34 & 1,421 & 1,797 & $0,190^{a b}$ \\
\hline 9 & 22 & 1,434 & $1,786^{\mathrm{ab}}$ & $0,202^{a}$ & 29 & 1,418 & 1,790 & $0,190^{\mathrm{ab}}$ \\
\hline 10 & 7 & 1,437 & $1,777^{\mathrm{ab}}$ & $0,202^{a}$ & 9 & 1,408 & 1,770 & $0,189^{a b}$ \\
\hline 11 & 16 & 1,439 & $1,787^{a b}$ & $0,201^{a b}$ & 3 & 1,435 & 1,800 & $0,188^{a b}$ \\
\hline 12 & 3 & 1,430 & $1,730^{b}$ & $0,200^{a b c}$ & - & - & - & - \\
\hline 13 & - & - & - & - & 2 & 1,430 & 1,750 & $0,195^{a}$ \\
\hline
\end{tabular}

Na coluna, médias com a letra diferente diferem estatisticamente pelo Teste de Tukey. 


\section{KURTZ e LÖF}

Nestas verificou-se que no parâmetro altura há diferença significativa entre potrancas menores (idade máxima de 37 meses) e éguas adultas (idade mínima de 50 meses), as fêmeas mais velhas possuem medidas maiores.

A confirmação desta tendência é observada na TABELA 3: animais mais velhos apresentarem uma altura maior, embora não exista diferença significativa deste parâmetro nas diferentes idades. Esta tendência não ocorre nos perímetros torácico e de canela, cujas médias são mais homogêneas. A comparação destas médias com aquelas determinadas pela associação de criadores do Brasil, demonstra que a altura encontra-se próxima do limite mínimo, porém os perímetros torácico e de canela estão dentro das médias recomendadas.

Trabalhos avaliando a placa de crescimento epifisiário em ossos relacionados com o crescimento em altura dos animais em diferentes raças demonstraram que: nas fêmeas da raça mangalarga a placa epifisiária distal do rádio cessa o seu crescimento aos 25 meses de idade (MAMPRIM et al., 1992); na raça brasileira de hipismo a placa epifisiária distal do rádio cessa seu crescimento nas fêmeas aos 25,8 meses e nos machos aos 28,16 meses (GODOY et al., 2004).

$\mathrm{Na}$ raça crioula o fechamento da placa epifisiária do osso metacárpico III ocorre dos seis a sete meses de idade; o fechamento da placa epifisiária da tíbia ocorre a partir dos 22 meses de idade; e a placa epifisiária distal do rádio cessa o seu crescimento a partir dos 22 meses (LUIZ, 2005). Assim, o crescimento em altura dos animais da raça crioula é interrompido ao redor dos 24 meses.

Embora o grupo estudado fosse de animais submetidos à rigorosa seleção e de alta qualidade zootécnica, apresentados no evento mais importante para a raça crioula e, conseqüentemente sob elevada dieta nutricional, os resultados do presente trabalho evidenciam uma tendência dos animais jovens apresentarem uma menor altura. É possível que a utilização de ascendentes de estatura baixa possa ser responsabilizada por esta característica. No Chile a Federação de Rodeio e Criadores de Cavalos Chilenos enfatiza que animais de estatura baixa teriam maior pureza racial (FEDERACIÓN DE CRIADORES DE CABALLOS CHILENOS, 2006) e seriam mais adaptados às modalidades esportivas eqüestres chilenas.

Trabalho realizado por PORTE (2000) em 67 cavalos chilenos com três anos de idade verificando as mesmas medidas aqui descritas, obteve: altura média de 1,374m (machos) e 1,377m (fêmeas), perímetro torácico médio de $1,661 \mathrm{~m}$ (machos) e 1,693m (fêmeas), e perímetro de canela médio de 0,182 (machos) e $0,179 m$ (fêmeas). Concluiu que a altura é o parâmetro a ser melhorado, e que os perímetros torácico e de canela apresentavam médias dentro do padrão recomendado.

A partir da década de 70 passou-se a utilizar garanhões chilenos com a intenção de melhorar a capacidade de trabalho do cavalo crioulo riograndense, e nas décadas seguintes houve um aumento na importação destes animais. Ressalvase que apesar desta grande utilização, existe ainda uma ampla variabilidade genética da população de cavalos crioulos (VINOCUR et al., 2003).

As médias de altura aqui observadas: $1,43 \mathrm{~m}$ (machos) e 1,42m (fêmeas) indicam que este parâmetro está próximo do limite mínimo e, é a medida sobre o qual se deve ter maior atenção. Com relação ao perímetro torácico e perímetro de canela a tendência a medidas maiores em animais mais velhos não se confirma, e provavelmente seja a manifestação de característica não relacionada com a altura.

\section{CONCLUSÕES}

Os resultados obtidos permitem concluir que os parâmetros analisados: altura perímetros torácico e de canela estão dentro dos limites determinados pela associação de criadores e que há uma tendência dos animais mais jovens apresentarem uma altura menor do que a dos mais velhos.

\section{AGRADECIMENTOS}

Os autores agradecem ao Sr. Edilon Xavier de Almeida, chefe do serviço de registro de genealógico da $A B C C C$ pela disponibilização dos dados e ao Professor Dr. José Henrique Souza da Silva pelo auxílio na análise estatística.

\section{REFERÊNCIAS}

ASSOCIAÇÃO BRASILEIRA DE CRIADORES DE CAVALOS CRIOULOS. Regulamento do registro genealógico da raça crioula. Disponível em: $<$ http://www.abccc.com.br/p regulamento.php?e_p=08>. Acesso em: 05 abr. 2006. 
Biometria de eqüinos da raça crioula no brasil.

ASSUNÇÃO,F.O. El caballo criollo. Buenos Aires: EMECE, 2000.99p.

CABRAL,G.C.; ALMEIDA,F.Q.de; QUIRINO,C.R.; PINTO,L.F.B.; SANTOS,E.M.; CORASSA,A. Avaliação morfométrica de eqüinos da raça mangalarga marchador: medidas lineares. Revista Brasileira de Zootecnia, v.33, n. 4, p.989-1000, 2004.

DOWDALL,R.C. Criando criollos. Montevideo: Hemisferio Sur, 1985.409p.

DOWDALL,C.R. Caballo criollo, el caballo del país. Buenos Aires: Vasquez Mazzini, 2003. 271p.

FEDERACIÓN DE CRIADORES DE CABALLOS CHILENOS. ¿Qué es sello de raza? Disponível em: $<$ http://200.75.0.7/Rodeo/controlador/controlador/control. jsp?ACCION=general/general\&SUBACCION=QueEsEIS elloDeRaza>. Acesso em: 30 mar. 2006.

GODOY,C.L.B.; VULCANO,L.C.; SANTOS,F.A.M.; SOARES,J.C.M. Fechamento epifisário da extremidade distal do rádio de eqüinos da raça brasileira de hipismo. Ciência Rural, v.34, n.6, p.1813-1815, 2004.

LUIZ, R. C. Anatomia radiológica da placa de crescimento dos ossos longos em potros crioulos. Santa Maria, 2005. 31p. Dissertação (Mestrado em Medicina Veterinária), Programa de Pós-Graduação em Medicina Veterinária, Universidade Federal de Santa Maria.
MAMPRIM,M.J.; VULCANO,L.C.; MUNIZ,L.M.R. Estudo radiográfico do fechamento da epífise distal do rádio em potros da raça manga-larga. Veterinária e Zootecnia, v.4, p.59-62, 1992.

MCMANUS,C.; FALCÃO,R.A.; SPRITZE,A.; COSTA,D.; LOUVANDINI,H.; DIAS,L.T.; TEIXEIRA,R.deA.; REZENDE,M.J.deM.; GARCIA,J.A.S. Caracterização morfológica de eqüinos da raça campeiro. Revista Brasileira de Zootecnia, v.34, n. 5, p.1153-1562, 2005.

MISERANI,M.G; McMANUS,C.; SANTOS,S. A.; SILVA,J.A.da; MARIANTE, A.daS.; ABREU,U.G.P.de. Avaliação dos fatores que influem nas medidas lineares do cavalo pantaneiro. Revista Brasileira de Zootecnia, v.31, n. 1, p.335-341, 2002.

PORTE, E. Crecimiento e desarrollo del caballo criollo chileno. Avances en Producción Animal, v.25, n.1 e 2, p.167-177, 2000.

SAS, Institute. SAS user's guide. Cary, 1999. 295p.

VINOCUR, M.E.; BRASS,K.E.; RUBIN,M.I.B.; SILVA,C.A.M. Genetic variability in the brazilian criollo horse breed. Ciência Rural, v.133, n.1, p.137-142, 2003. 